\title{
A Statistical Index for Evaluating the CTV Coverage Loss of Brain Stereotactic Radiosurgery When the PTV Margin Is Zero
}

\author{
Jenghwa Chang1,2 \\ ${ }^{1}$ Department of Radiation Medicine, Northwell Health and Donald and Barbara Zucker School of Medicine at Hofstra/Northwell, \\ New York, USA \\ ${ }^{2}$ Department of Physics and Astronomy, Hofstra University, New York, USA \\ Email: jchang24@northwell.edu
}

How to cite this paper: Chang, J. (2022) A Statistical Index for Evaluating the CTV Coverage Loss of Brain Stereotactic Radiosurgery When the PTV Margin Is Zero. International Journal of Medical Physics, Clinical Engineering and Radiation Oncology, 11, 84-97.

https://doi.org/10.4236/ijmpcero.2022.111008

Received: November 1, 2021

Accepted: February 25, 2022

Published: February 28, 2022

Copyright $\odot 2022$ by author(s) and Scientific Research Publishing Inc. This work is licensed under the Creative Commons Attribution International License (CC BY 4.0).

http://creativecommons.org/licenses/by/4.0/

\begin{abstract}
Purpose: To develop a new statistical index "percent CTV (clinical target volume) coverage probability" (\%CCP), defined as the probability that a specific percent (e.g., 95\%) of the CTV can be reliably covered by the prescription dose, for evaluating the coverage loss of brain (fractionated) stereotactic radiosurgery (SRS/fSRS) when the PTV (planning target volume) margin is zero. Methods: The random variable $Q$ for CTV percent coverage was derived using a previously developed model for CTV random motion that follows a three-dimensional (3D) independent normal distribution with a zero mean and a standard deviation of $\sigma_{S}$ (for translation) or $\sigma_{\delta}$ (for rotation). Assuming both CTV and PTV are spherical with the same diameter $d_{\mathrm{CTV}}$, the cumulative distribution function of $Q$ could be obtained analytically using the relation of spheresphere intersection. The \%CCP was then derived as the reliability function of $Q$ and was used to quantify the coverage loss for selected $d_{\text {Стv }}$. Results: The $95 \%$ - $95 \%$ clinical goal (95\% of the times, at least $95 \%$ of the CTV is covered) is not achievable with $d_{\mathrm{CTV}}<42 \mathrm{~mm}$. For common CTVs $\left(d_{\mathrm{CTV}}<20 \mathrm{~mm}\right)$ encountered in SRS/fSRS, only $60 \%-90 \%$ of the CTV could be reliably covered by the prescription dose $95 \%$ of the time. For $\sigma_{S}=0.5 \mathrm{~mm}$ and $\sigma_{\delta}=0.4^{\circ}$, the 95\% CCP was the highest when the distance between the CTV and the isocenter $d_{I \Leftrightarrow T}=0$ and gradually decreased with the increasing $d_{I \Leftrightarrow T}$. Conclusions: The \%CCP was successfully derived for evaluating the CTV coverage loss for brain SRS/fSRS. When the PTV margin is zero, the 95\% - 95\% clinical goal cannot be achieved for most targets $\left(d_{\mathrm{CTV}}<42 \mathrm{~mm}\right)$.
\end{abstract}

\section{Keywords}

Stereotactic Radiosurgery, PTV Margin, Coverage Probability 


\section{Introduction}

Brain Stereotactic Radiosurgery (SRS) was the first radiotherapy procedure that employed very high-precision immobilization/setup devices, e.g., frame [1] [2] and recently IGRT (image-guided radiotherapy) [3] [4] [5] [6] systems, for treating small brain targets with a high radiation dose delivered in one fraction. This concept of SRS was later extended to the Fractionated Stereotactic Radiosurgery (fSRS), which allows up to five-fraction treatments using similar immobilization devices with comparable or slightly inferior setup accuracy. The accuracy of these immobilization/setup devices, including the apparatus accuracy and total accuracy, has been thoroughly investigated [5] [6] [7] [8] [9]. Almost all studies demonstrated sub-millimeter apparatus accuracy, although the total accuracy generally ranges between 1 and 3 millimeters, depending on the uncertainties introduced through the whole treatment process. Based on the analysis of total accuracy, an expansion margin on the order of 1-2 mm around the Clinical Target Volume (CTV) is recommended for generating the Planning Target Volume (PTV) to achieve the desired "CTV coverage probability", that is, $100 \%$ of the CTV is covered by the prescription dose for a specified probability (e.g., $95 \%$ of the time) [10].

However, because of the high apparatus accuracy, it is a common practice in some centers using the CTV as the PTV (i.e., with a zero PTV expansion margin) for brain SRS/fSRS. For example, in the RTOG Protocol 90-05 for brain SRS [11], "the target volume was defined as the contrast enhancing tumor volume without a margin of surrounding brain tissue". This approach essentially ignores all uncertainties in the treatment process and assumes the apparatus is perfect. Since no treatment process and apparatus are flawless, the CTV might not be covered by the prescription dose as expected when the PTV margin is 0. Even so, the actual coverage loss is not well quantified. As will be explained in the "Methods" Section, the CTV coverage probability becomes zero when the PTV margin is zero. This is true even only a small fraction of the CTV is not covered by the prescription dose. Therefore, the CTV coverage probability is not an appropriate index for evaluating how well the CTV is covered when the PTV margin is zero, and an alternative index needs to be developed.

In this study, a new statistical index, the "percent CTV coverage probability" (\%CCP) defined as the probability of a specific percent (e.g., 95\%) of the CTV can be reliably covered by the prescription dose (or equivalently the PTV), was proposed to evaluate the coverage loss of brain SRS/fSRS when the PTV margin is zero. To calculate this probability, a previously developed statistical model [12] was used to analyze the CTV random motion, from which the random variable $Q$ for CTV percent coverage was derived for measuring the random loss of CTV percent coverage when the PTV margin is 0 . The percent CTV coverage probability is therefore equal to the reliability of $Q$ for the specified percent CTV coverage.

In general, the closed-form expressions for $Q$ and the percent CTV coverage 
probability do not exist. However, for perfectly spherical targets, the formulas of both quantities can be derived analytically using the relation of sphere-sphere intersection [13]. As pointed out in the report of RTOG 90-05 [11], most targets treated with SRS/fSRS can be approximated by a sphere or ellipse with a maximal diameter of $40 \mathrm{~mm}$ or less. The author, therefore, focused the analysis on spherical CTVs with a diameter $\left(d_{\mathrm{CTV}}\right)$ less than $40 \mathrm{~mm}$ to simplify the derivations of the cumulative distribution function and the reliability function of $Q$.

In this paper, the author will first present the definitions of $Q$ and \%CCP. The Cumulative Distribution Function (CDF) and reliability function of $Q$ for perfectly spherical targets will then be derived analytically from the Probability Density Function (PDF) of the random vector for CTV motion. Finally, the \%CCP for clinically interested (e.g., the $95 \%$ - $95 \%$ clinical goal or $95 \%$ of the CTV covered by the prescription dose at least $95 \%$ of the time) cases will be shown for selected CTV sizes and distances from the isocenter. Clinical implications of these results and the applications of the percent CTV coverage probability will be discussed.

\section{Methods and Materials}

\subsection{The Statistical Model}

The author has developed a statistical model for analyzing the combined translational and rotational error of the CTV random motion relative to the treatment isocenter [12]. Details of this model was described in an early publication [12]. In brief, let $\mathbf{S}$ and $\mathbf{R}$ denote the random vectors for the translational and rotational errors in the three-dimensional (3D) patient coordinate system and $\mathbf{E}=\mathbf{S}+\mathbf{R}$ be the random vector for the combined setup error. Assuming both $\mathbf{S}$ and $\mathbf{R}$ follow the $3 \mathrm{D}$ independent normal distribution with a zero mean and a uniform standard deviation of $\sigma_{S}$ and $\sigma_{R}$, respectively, the probability density functions (PDFs) of $\mathbf{S}$ and $\mathbf{R}$ are:

$$
\begin{aligned}
& f_{\mathrm{S}}(x, y, z)=\frac{1}{\left(2 \pi \sigma_{S}^{2}\right)^{3 / 2}} \mathrm{e}^{-\frac{x^{2}+y^{2}+z^{2}}{2 \sigma_{S}^{2}}} . \\
& f_{\mathrm{R}}(x, y, z)=\frac{1}{\left(2 \pi \sigma_{R}^{2}\right)^{3 / 2}} \mathrm{e}^{-\frac{x^{2}+y^{2}+z^{2}}{2 \sigma_{R}^{2}}} .
\end{aligned}
$$

Since $\mathbf{S}$ and $\mathbf{R}$ are independent random variables, the combined error $\mathbf{E}$ also follows a $3 \mathrm{D}$ normal distribution with a zero mean and a uniform standard deviation of $\sigma_{E}=\sqrt{\sigma_{S}^{2}+\sigma_{R}^{2}}$ :

$$
f_{\mathrm{E}}(x, y, z)=\frac{1}{\left(2 \pi \sigma_{E}^{2}\right)^{3 / 2}} \mathrm{e}^{-\frac{x^{2}+y^{2}+z^{2}}{2 \sigma_{E}^{2}}} .
$$

Applying change of variables $u_{x}=\frac{x}{\sigma_{E}}, u_{y}=\frac{y}{\sigma_{E}}, u_{z}=\frac{z}{\sigma_{E}}$ in Equation (2) and transforming the PDF from the Cartesian to the spherical coordinates, the PDF 
$f_{P}(\rho)$ for the random motion $P$ in the radial direction can be derived as:

$$
f_{P}(\rho)=\frac{2}{\sqrt{2 \pi}} \rho^{2} \mathrm{e}^{-\frac{\rho^{2}}{2}},
$$

which is the chi distribution with three degrees of freedom (3 DOF). A further change of variable $u=\rho^{2}$ leads to:

$$
f_{P^{2}}(u)=\frac{1}{\sqrt{2 \pi}} \sqrt{u} \mathrm{e}^{-\frac{u}{2}},
$$

which is the chi-square distribution with $3 \mathrm{DOF}$.

\subsection{CTV Coverage Loss When the PTV Margin Is 0}

The purpose of this study was to investigate how much the CTV coverage is lost when the PTV margin is 0 . To achieve this goal, a statistical index needed to be identified first to quantify the CTV coverage loss. Traditionally, the CTV coverage is evaluated using the "CTV coverage probability", that is, the probability the CTV is still covered by the prescription dose (or PTV) when the setup error is present. The CTV coverage probability is a very good index for quantifying the effectiveness of a non-zero PTV margin. For a given PTV margin, the CTV coverage probability is $100 \%$ when there is no setup uncertainty, i.e., $\sigma_{E}=0$ in Equation (2), and gradually decreases with increasing $\sigma_{E}$. The effectiveness of a non-zero PTV margin can therefore be evaluated by examining if the coverage probability will remain above a desired value, typically $95 \%$ of the time, for the setup error encountered in clinics.

However, when the PTV margin is 0 , the CTV will not be fully covered by the prescription dose unless the magnitude of CTV random motion E is 0 , or $\rho=0$ in Equation (3). Since the chi distribution in Equation (3) is a continuous function, the probability for $\rho=0$ is 0 unless $\sigma_{E}=0$ in Equation (2). Given that the setup is not perfect, $\sigma_{E}$ in Equation (2) is always a positive real number and is not equal to zero. As a result, the probability of full CTV coverage by the prescription dose is always $0 \%$ if the PTV margin is 0 .

Therefore, the CTV coverage probability (by the prescription dose) is not ideal for quantifying the CTV coverage loss when the PTV margin is 0 and an alternative index is needed. This new index must have a reasonable probability distribution, that is, its probability is $100 \%$ when there is no setup error or $\sigma_{E}=0$ in Equation (2), gradually decreases with increasing $\sigma_{E}$, and approximate 0 for large $\sigma_{E}$.

\subsection{Percent CTV Coverage Probability and CTV Percent Coverage Random Variable}

One concept similar to the CTV coverage probability, termed as the "percent CTV coverage probability" or \%CCP in this study is the probability that at least a certain percent (e.g., 95\%) of the CTV is covered by the prescription dose, or equivalently, the probability that at most a complementary percent (e.g., 100\% - 
$95 \%=5 \%)$ of the CTV is not covered by the prescription dose. This \%CCP can be derived from the reliability function of the random variable $Q$ for quantifying the CTV percent coverage as a function of CTV random motion E:

$$
Q=\frac{V_{\mathrm{C} \cap \mathrm{P}, \mathrm{E}}}{V_{\mathrm{C}}}
$$

where: $V_{\mathrm{C}}$ is the volume of CTV, and $V_{\mathrm{C} \cap \mathrm{P}, \mathrm{E}}$ is the volume of PTV $\cap \mathrm{CTV}$ or the CTV still covered by the PTV after the random motion E. Note that since $\mathrm{E}$ is a random vector, $Q$ is a scalar random variable. The cumulative distribution function $(\mathrm{CDF})$ of $Q$ is $F_{Q}(q)=\operatorname{Prob}(Q<q)$. Given that the 3D normal distribution of $\mathbf{E}$ in Equation (2) is circularly symmetric, $F_{Q}(q)$ can be derived from the CDF of random variable $P^{2}$ for CTV random motion in Equation (4). The reliability function of $Q$ is therefore $S_{Q}(q)=\operatorname{Prob}(Q>q)=1-F_{Q}(q)$, and the \%CCP for a chosen $100 q_{0} \%$ (e.g., $95 \%$ when $q_{0}=0.95$ ) CTV coverage is $S_{Q}\left(q_{0}\right)$.

The percent CTV coverage probability defined above is a more appropriate index when the PTV margin is 0. Because a fraction (e.g., 95\%) of the CTV is smaller than the PTV, there is a finite probability that the specified percent CTV might still be covered by the PTV when the CTV random motion occurs. Therefore, the \% CCP is not always 0 but a number between 0 and 1, depending on the specified "percent coverage" and the values of $\sigma_{E}$ for the CTV random motion. For a chosen percent CTV, the coverage probability is $100 \%$ if $\sigma_{E}=0$, and gradually decreases with increasing $\sigma_{E}$.

The chosen $q_{0}$ for quantifying the \%CCP must make clinical sense. Clinically, a $90 \%-100 \%$ coverage of the treatment target volume is generally acceptable when evaluating the radiotherapy treatment planning. Note that the percent CTV coverage probability reduces to the traditional CTV coverage probability if $100 \%$ CTV coverage is specified. Therefore, a value of $q_{0}$ close to $100 \%$ will make $F_{Q}(q)$ approximate a degenerate CDF like the one for CTV coverage probability which, as discussed earlier, is not ideal for quantifying the CTV coverage loss when the PTV margin is 0 . In this study, 95\% CTV coverage (i.e., $q_{0}=0.95$ ) was chosen as the evaluation criterion.

\subsection{Derivation of $F_{Q}(q)$ for Perfectly Spherical Cases}

The CDF of $Q, F_{Q}(q)$ is a function of CTV random motion and the shape of CTV. Although the CDF of $P^{2}$ for CTV random motion is known in Equation (4), the calculation of percent coverage in Equation (5) is shape specific and generally cannot be solved analytically.

In this study, the author focused on the random motion and percent coverage for perfectly shaped spherical CTVs. This ideal shape was chosen because the closed-form expression of $F_{Q}(q)$ exists if the CTV is perfectly spherical. In addition, quasi-sphered CTVs are frequently encountered in brain SRS/fSRS [11] [14]. When the PTV margin is 0 , the shape of PTV is also spherical with the same 
radius because its shape is the same as that of CTV.

Figure 1 illustrates the random motion of CTV relative to the PTV. In Figure 1, both CTV and PTV are spherical and have the same radius $r_{\mathrm{P}}=r_{\mathrm{C}}=r$ because the PTV margin is 0 . Due to the CTV random motion E, the centers of CTV and PTV do not coincide and the square of the distance $\rho$ between these two centers is, as described above, an event of the random variable $P^{2}$ following the chisquare distribution with 3 DOF. In Figure 1, the area shaded in blue is $V_{\mathrm{C} \cap \mathrm{P}, \mathrm{E}}$ in Equation (5), which denotes the volume of the intersection of CTV and PTV (i.e., $\mathrm{C} \cap \mathrm{P}$ ) when separated by distance $\rho$, or the part of CTV still covered by the prescription dose cloud (i.e., PTV).

The ratio of $V_{\mathrm{C} \cap \mathrm{P}, \mathrm{E}}$ to the total CTV volume, $\frac{V_{\mathrm{C} \cap \mathrm{P}, \mathrm{E}}}{V_{\mathrm{C}}}$ is the percent of CTV that is still covered by the prescription dose after the random motion $\mathrm{E}$ and can be solved analytically. That is, using the relation of sphere-sphere intersection [13], the closed-form expression of $V_{\mathrm{C} \cap \mathrm{P}, \mathrm{E}}$ is:

$$
V_{\mathrm{C} \cap \mathrm{P}, \mathrm{E}}=2 \pi\left(r-\frac{\rho}{2}\right)^{2}\left[r-\frac{1}{3}\left(r-\frac{\rho}{2}\right)\right]=\frac{1}{12} \pi r^{3}\left(2-\frac{\rho}{r}\right)^{2}\left(4+\frac{\rho}{r}\right) .
$$

The CTV percent coverage is therefore equal to $V_{\mathrm{C} \cap \mathrm{P}, \mathrm{E}}$ divided by the volume of CTV, $V_{\mathrm{C}}=\frac{4}{3} \pi r^{3}$ :

$$
\frac{V_{\mathrm{C} \cap \mathrm{P}, \mathrm{E}}}{V_{\mathrm{C}}}=\frac{1}{16}\left(2-\frac{\rho}{r}\right)^{2}\left(4+\frac{\rho}{r}\right)
$$

Note that $\frac{V_{\mathrm{C} \cap \mathrm{P}, \mathrm{E}}}{V_{\mathrm{C}}}$ is also the output of a random variable, because $\rho$ is the output of a chi random variable $P$. Therefore, the random variable $Q$ for percent

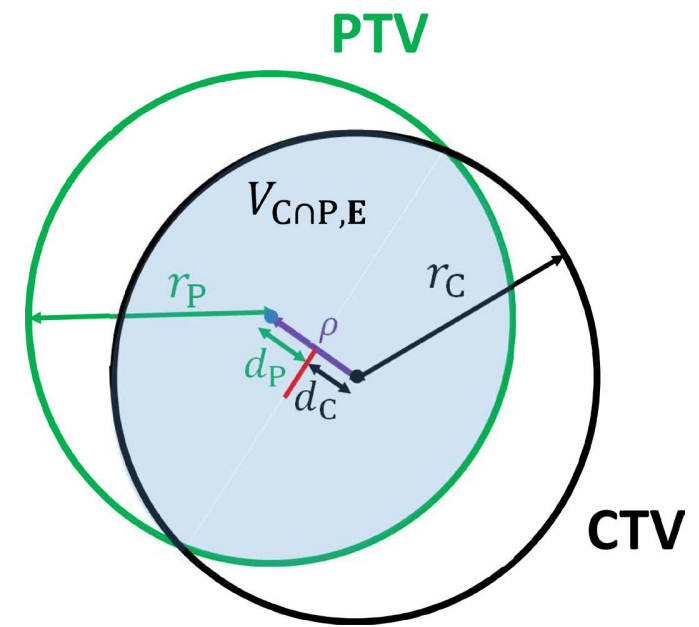

Figure 1. Illustration of the CTV random motion relative to the PTV assuming both CTV and PTV are spherical with the same radius $r_{\mathrm{P}}=r_{\mathrm{C}}=r$. The area shaded in blue, $V_{\mathrm{C} \cap \mathrm{P}, \mathrm{E}}$, denotes the part of CTV still covered by the prescription dose cloud (i.e., PTV) after the CTV random motion $\mathrm{E}$ that leads to a separation of $\rho$ between the centers of these two target volumes. 
coverage of a spherical CTV is a function of random variable $P$.

$$
Q=g\left(\frac{P}{r}\right)=\frac{1}{16}\left(2-\frac{P}{r}\right)^{2}\left(4+\frac{P}{r}\right)
$$

which quantifies the fraction of CTV remained inside the PTV after random motion $P$. Note that $Q$ is a monotonically decreasing function of $\frac{P}{r}$ for $0 \leq \frac{P}{r} \leq 2$. That is, $Q=1$ (a full coverage) when $\frac{P}{r}=0$, gradually decreases with increasing $\frac{P}{r}$ until $Q=0$ (a complete miss) when $\frac{P}{r}=2$. The $\mathrm{CDF}$ of $Q$ can be derived from the PDF of $P^{2}, f_{P^{2}}(u)=\frac{1}{\sqrt{2 \pi}} \sqrt{u} \mathrm{e}^{-\frac{u}{2}}$ :

$$
\begin{aligned}
F_{Q}(q) & =\operatorname{Prob}(Q \leq q)=\operatorname{Prob}\left(\frac{P}{r} \geq g^{-1}(q)\right)=\operatorname{Prob}\left(P \geq r g^{-1}(q)\right) \\
& =\operatorname{Prob}\left(P^{2} \geq\left(r g^{-1}(q)\right)^{2}\right)=\int_{\left(r g^{-1}(q)\right)^{2}}^{\infty} \frac{1}{\sqrt{2 \pi}} \sqrt{u} \mathrm{e}^{-\frac{u}{2}} \mathrm{~d} u
\end{aligned}
$$

\subsection{Calculation of the Reliability Function $S_{Q}(q)$}

As mentioned earlier, the goal of this study was to quantify the probability of CTV percent coverage (by the prescription dose) larger than a specified percent $q_{0}$ (e.g, $90 \%$ or $95 \%$ ), or $\operatorname{Prob}\left(Q>q_{0}\right)$. Statistically, this is equivalent to find $S_{Q}(q)$, the reliability or survival function of $Q$, from $F_{Q}(q)$, the CDF of $Q$ :

$$
S_{Q}(q)=\operatorname{Prob}(Q>q)=1-F_{Q}(q)=\int_{0}^{\left(r g^{-1}(q)\right)^{2}} \frac{1}{\sqrt{2 \pi}} \sqrt{u} \mathrm{e}^{-\frac{u}{2}} \mathrm{~d} u .
$$

In this study, $F_{Q}(q)$ in Equation (9) and $S_{Q}(q)$ in Equation (10) were programmed in a Microsoft Excel spreadsheet using the "CHISQ.DIST()" function for the chi-square distribution. The inverse function $g^{-1}(q)$ was calculated using the "Solver" function in Excel for finding optimal solutions for various kind of decision problems.

\subsection{Calculation of the $95 \%$ CTV Coverage Probability for the Single-Isocenter-for-Multiple-Target Technique}

The 95\% CTV coverage probability (95\% CCP) for the single-isocenter-formultiple-target technique was calculated for a set of commonly encountered clinical parameters, $\sigma_{S}=0.5 \mathrm{~mm}$ and $\sigma_{\delta}=0.4^{\circ}$, as a function of the distance between the CTV and isocenter, $d_{I \Leftrightarrow T}$. For a $d_{I \Leftrightarrow T}, \sigma_{\delta}$ was first converted to $\sigma_{R}$ using the relation $\sigma_{R}=0.816 d_{I \Leftrightarrow T} \sigma_{\delta} \frac{\pi}{180}$ as described in the previous publication [12]. $\sigma_{E}$ is then calculated as $\sigma_{E}=\sqrt{\sigma_{S}^{2}+\sigma_{R}^{2}}$, from which the 95\% CCP was obtained for various CTV size using the method described above. 


\section{Results}

Figure 2 shows the CDF of random variable $Q$ for the CTV percent coverage (by the PTV), for CTVs of various radii $r$ normalized to $\sigma_{E}$. For example, "1" means $r=\sigma_{E}$ and " 50 " $r=50 \sigma_{E}$. If $\sigma_{E}=0.5 \mathrm{~mm}$, "1" means $r=0.5 \mathrm{~mm}$ (or diameter $=1 \mathrm{~mm}$ ) and " 50 " $r=25 \mathrm{~mm} \mathrm{(or} \mathrm{diameter}=50 \mathrm{~mm}$ ). Note that $F_{Q}(q)=\operatorname{Prob}(Q \leq q)$ is the cumulative probability of less than $100 q \%$ (or between $0 \%$ and $100 q \%$ ) of CTV covered by the PTV. The probability for a complete miss is the intersection of a curve with the $\mathrm{Y}$-axis (or when $Q=0$ ) in Figure 2.

Figure 3 illustrates the reliability function $S_{Q}(q)$, or the probability of $Q$ larger than a given value $q$. Again, the various CTV sizes $r$ are normalized to $\sigma_{E}$ as in Figure 2. The vertical red dotted line indicates $q=q_{0}=0.95$, or the reliability threshold for $95 \%$ CTV coverage. Intersection of this vertical line with a curve marks $S_{Q}(0.95)$, or the $95 \%$ CCP for that CTV size. The horizontal red dotted lines indicate the $95 \%$ reliability threshold for the CTV percent coverage. Intersection of this horizontal line with a curve specifies the value of $Q$ or the percent of CTV that can be reliably covered by the PTV $95 \%$ of the time for that CTV size. The red-shaded area in the upper right corner is the "95\% - 95\% clinical goal" adopted in this study, that is, any curve entering this zone indicates the CTV of that size has a $95 \%$ probability that $95 \%$ or more of the CTV is covered by the PTV.

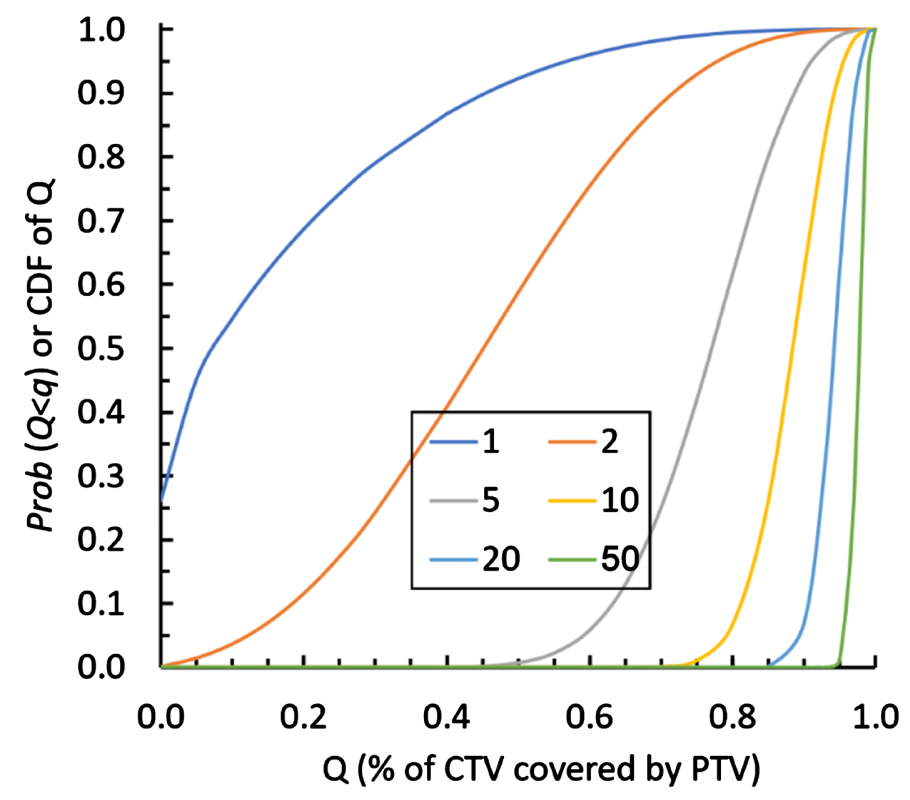

Figure 2. Cumulative Distribution Function (CDF) of random variable $Q, F_{Q}(q)$ for CTV percent coverage (by the PTV) for various radii $r$ normalized to $\sigma_{E}$ (e.g., "1" means $r=1 \sigma_{E}$ and " $\left.50 " \quad r=50 \sigma_{E}\right) . \quad F_{Q}(q)=\operatorname{Prob}(Q \leq q)$ is the cumulative probability of less than $100 q \%$ (or between $0 \%$ and $100 q \%$ ) of CTV covered by the PTV. The probability for a complete miss or $Q=0$ is negligible (i.e., $\sim 0$ probability) except for $r=1 \sigma_{E} \quad($ Prob $=0.26)$. 


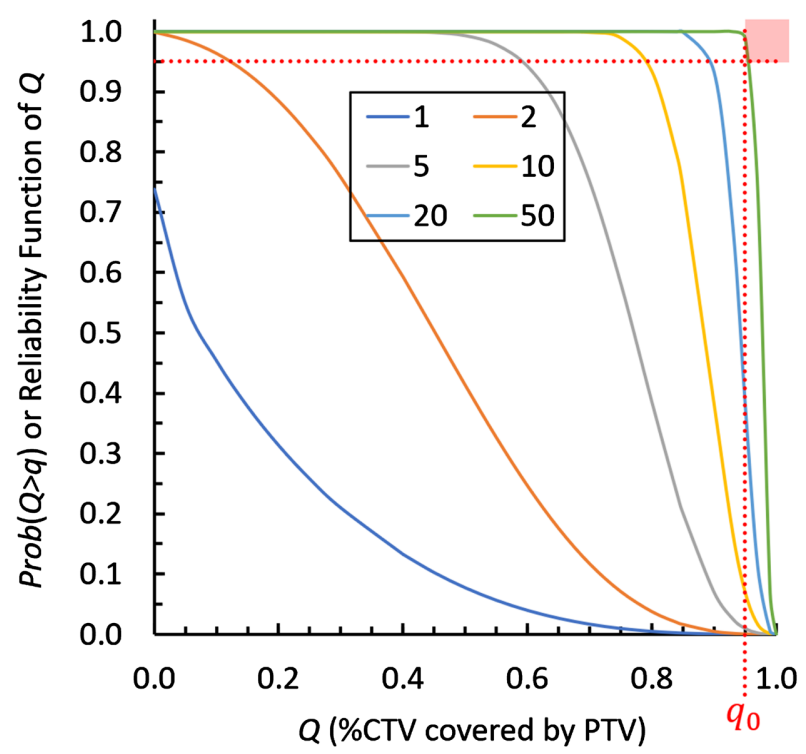

Figure 3. Reliability function of random variable $Q, S_{Q}(q)$ for CTV percent coverage (by the PTV) for various radii $r$ normalized to $\sigma_{E}$ (e.g., "1" means $r=\sigma_{E}$ and " 50 " means $\left.r=50 \sigma_{E}\right) . S_{Q}(q)=\operatorname{Prob}(Q>q)$ is the probability of $Q$ larger than a given value $q$. The vertical and horizontal dashed red lines indicate the $95 \%$ reliability thresholds for respectively $q$ and $S_{Q}(q)$, the functions of which are explained in detail in the text.

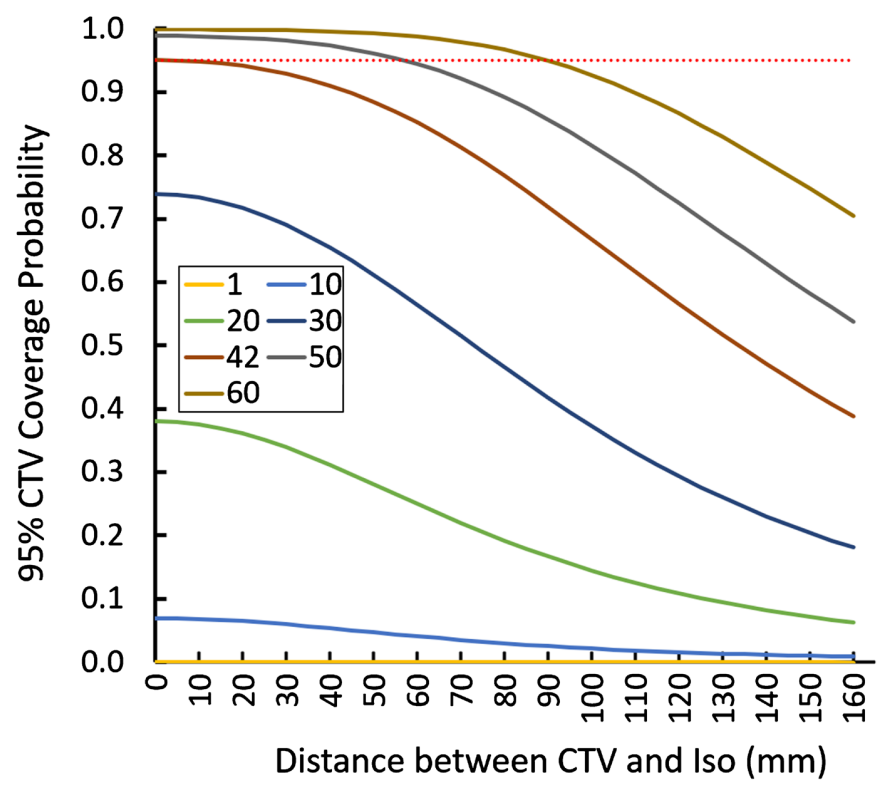

Figure 4. The 95\% CTV coverage probability $(95 \% \mathrm{CCP})$ for $\sigma_{S}=0.5 \mathrm{~mm}$ and $\sigma_{\delta}=0.4^{\circ}$ as a function of the distance from the CTV to the isocenter, for various CTV diameters $\left(d_{\text {CTV }}\right)$ ranging from $1 \mathrm{~mm}$ to $60 \mathrm{~mm}$ (e.g., "1" means diameter $=1 \mathrm{~mm}$ and " 50 " means diameter $=50 \mathrm{~mm}$ ). The dashed red line indicates the $95 \%$ reliability preference.

Figure 4 shows the 95\% CCP for $\sigma_{S}=0.5 \mathrm{~mm}$ and $\sigma_{\delta}=0.4^{\circ}$. This figure is particularly relevant to the single-isocenter-for-multiple-target technique because it is plotted as a function of the distance between the CTV and isocenter, $d_{I \Leftrightarrow T}$ in 
millimeter, for various CTV diameters $\left(d_{\text {СтV }}\right)$ ranging from $1 \mathrm{~mm}$ to $60 \mathrm{~mm}$ (e.g., "1" means $d_{\text {СТV }}=1 \mathrm{~mm}$ and " 50 " means $d_{\text {СTV }}=50 \mathrm{~mm}$ ). The dashed red line indicates the $95 \%$ reliability preference. Note that all distances in this figure are in unit of millimeter instead of being normalized to $\sigma_{E}$ as in the previous two figures. When $\sigma_{E}=0.5 \mathrm{~mm}$ in Figure 2 and Figure 3, the curves marked by the same numerical value have the same size in Figures 2-4. For example, for the curves marked by " 20 " in Figure 2 or Figure 3 have a radius of $10 \mathrm{~mm}$, or a diameter of $20 \mathrm{~mm}$, which is the size for the curved marked by "20" in Figure 4 .

\section{Discussion}

In this study a new statistical index, the percent CTV coverage probability (or \%CCP) was developed for evaluating the CTV coverage loss of brain SRS/fSRS when the PTV margin is zero. The motivation for developing this new index is because there is an intrinsic deficiency in the CTV coverage probability traditionally used for this purpose. That is, although very effective in evaluating the CTV coverage loss for a non-zero PTV margin, the CTV coverage probability is a degenerate function (i.e., always 0 ) when the PTV margin is 0 . The proposed \%CCP, on the other hand, is a more meaningful index because its output is a reasonable probability between 0 and 1 unless the selected percent CTV coverage is close to $100 \%$. Since $90 \%-100 \%$ CTV coverage is clinically acceptable, the $\%$ CCP is reasonable once the specified percent CTV coverage is not too close to $100 \%$ (e.g., $95 \%$ ), as proven by the results presented in this paper.

The main disadvantage of the proposed \%CCP is that its analytic form usually does not exist except for special geometries like the spherically-shaped targets adopted in this study. This disadvantage is not unique to the \%CCP, but is common to other similar indexes, e.g., the traditional CTV coverage probability, that use statistical modeling to analyze the CTV random motion. A general approach to solve the statistical modeling problems is performing Monte Carlo simulations for each specific geometry, which can be time-consuming and sometimes impractical. Fortunately, most targets treated with SRS/fSRS can be approximated by a sphere or ellipse with a maximal diameter of $40 \mathrm{~mm}$ or less [11] [14]. Therefore, the proposed \%CCP can be used to predict the percent CTV coverage lost for most commonly encountered cases in SRS/fSRS.

To calculate the \%CCP, a previously published statistical model [12] was used to analyze the random motion of spherical CTVs. The basic assumptions of this statistical model [12] are that the translational, rotational and combined setup errors are isotropic, and are independent random variables following the normal distribution. This study was limited to spherical targets only because the CTV percent coverage by the PTV for a given setup error can be quantified analytically using the relation of sphere-sphere intersection [13]. In addition, most small SRS/fSRS targets encountered clinically tend to be roughly spherical in shape [11] [14] and therefore can be reasonably approximated by spheres of similar sizes. With these assumptions, the $\mathrm{CDF}$ and reliability function of the random variable $Q$ for 
CTV percent coverage were successfully derived from the chi-square distribution of the random variable $P^{2}$ for CTV random motion, and the numerical values of both functions were calculated in an MS Excel spreadsheet and plotted (Figures 2-4).

Figures 2-4 can be used to estimate the CTV percent coverage loss for brain SRS/fSRS procedures that use zero PTV margin. One interesting observation in Figure 2 is that the CDF curve for $r=1 \sigma_{E}$ has a non-zero ( $\left.\sim 0.26\right)$ value, indicating that there is a significant possibility that the CTV might be missed completely if the radius of CTV is on the order of the combined setup uncertainty $\sigma_{E}$. Assuming $\sigma_{E}=0.5 \mathrm{~mm}$, this result shows that there is a $\sim 26 \%$ chance that the treatment might miss the target completely if the CTV is $\sim 1 \mathrm{~mm}$ in diameter $\left(d_{\mathrm{CTV}}=1 \mathrm{~mm}\right)$. The probability of complete miss decreases quickly with increasing radius of the CTV, which is essentially 0 when $r \geq 2 \sigma_{E}$, as shown in Figure 2. The author would like to point out that a complete miss might happen in theory but not in practice. This is because for very small targets (on the order of a few millimeters in diameter), the CTV is usually contoured more generously and when the treatment plan is being developed, the planner usually makes the prescription isodose line less tight (around the CTV) purposely than that for larger targets.

Figure 3 sheds more lights on the \%CCP for a spectrum of target sizes. In Figure 3, the $q$ value of the intersection of the horizontal red dotted line with an $r$ curve indicates that the $100 q \%$ CTV coverage probability is $95 \%$, or $100 q \%$ or more of CTV will be covered by the prescription dose $95 \%$ of the time. The $S_{Q}(q)$ value of the intersection of the vertical red dotted line with an $r$ curve, on the other hand, is the probability of at least $95 \%$ of that CTV will be covered by the prescription dose. Any curve that enters the red shaded area at the upper right corner of Figure 3 will therefore have at least 95\% of its CTV covered by the prescription dose $95 \%$ of the time, which is the common "95\% - 95\%" clinical preference. If other threshold values for $q$ and $S_{Q}(q)$ are preferred, the locations of vertical and horizontal dotted lines should be adjusted.

It is observed in Figure 3 that the 95\% - 95\% clinical preference "at least 95\% of CTV covered by the prescription dose $95 \%$ of the chance" is not easily achievable as most curves do not enter the red shaded area except for $r=50 \sigma_{E}$. Assuming $\sigma_{E}=0.5 \mathrm{~mm}, r=50 \sigma_{E}$ corresponds to $d_{\mathrm{CTV}}=50 \mathrm{~mm}$. In fact, the minimal CTV size that will meet this preference is $r=42 \sigma_{E}=21(\mathrm{~mm})$ or $d_{\mathrm{CTV}}=42$ $\mathrm{mm}$, which is usually on the larger side of the targets for single fraction SRS. Common sizes for single fraction SRS are between $d_{\mathrm{CTV}}=10 \mathrm{~mm}$ and $d_{\mathrm{CTV}}=40$ $\mathrm{mm}$ [11] (or $5 \mathrm{~mm}$ and $20 \mathrm{~mm}$ in radius), corresponding to the " 5 ", "10" and " 20 " curves in Figure 3. Within this range, only between $60 \%$ (" 5 " curve) and $90 \%$ (" 20 " curve) of the CTV can be reliably covered by the prescription dose $95 \%$ of the time.

For the single-isocenter-for-multiple-target technique, $\sigma_{E}$, the standard deviation of the combined (translational and rotational) setup error, might not be a 
constant for all targets treated at the same time. Although the translational error is the same for all targets, the rotational error increases with $d_{I \Leftrightarrow T}[12]$. As a result, the percent CTV coverage probability might not be a constant but varies with $d_{I \Leftrightarrow T}$. This is evidenced in Figure 4 where the 95\% CTV coverage is plotted as a function of $d_{I \Leftrightarrow T}$ for various $d_{\mathrm{CTV}}$. Note that in Figure 4 , the clinical preference " $95 \%$ of the CTV covered by the prescription dose $95 \%$ of the time" is met only for the part of a curve that is above the red dotted line. When the geometric center of each target is positioned at the radiation isocenter for treatment, i.e., $d_{I \Leftrightarrow T}=0$ in Figure 4, the rotational error is insignificant and $\sigma_{E}$ is equal to the standard deviation of the translational error, or $\sigma_{E}=\sigma_{S}$. The 95\% CTV coverage probability for a CTV size is the same as the $S_{Q}(0.95)$ for the same CTV size, or the $S_{Q}$ value of the intersection of the red dotted line with the curve for that CTV size in Figure 3 (note that the CTV size is presented as the CTV radius normalized to $\sigma_{E}$ in Figure 3, and as CTV diameter in mm in Figure 4). For the single-isocenter-for-multiple-target technique, the target is not positioned at the radiation isocenter but at a distance $d_{I \Leftrightarrow T}$ from the isocenter. As illustrated in Figure 4, the 95\%CCP decreases with increasing $d_{I \Leftrightarrow T}$ for all CTV sizes. This is because a larger $d_{I \Leftrightarrow T}$ will lead to a larger

$\sigma_{R}\left(=0.816 d_{I \Leftrightarrow T} \sigma_{\delta} \frac{\pi}{180}\right)$, which results in a larger combined setup uncertainty $\sigma_{E}$ and therefore a lower 95\% CCP. Therefore, this model predicted that for single-isocenter for multiple-targets technique, the \%CCP will be lower for those targets farther away from the isocenter if the PTV margin is 0 for all targets.

Even though the 3D Gaussian random motion has been widely used to model the treatment uncertainty, it still needs to be validated for the specific studies it is applied to. Verification of this model is not in the scope of the current paper but will be performed in future studies. The author plans to use either Monte Carlo simulations and/or real clinical data to validate the theoretical calculations and predictions presented in this paper. The results will be presented in future publications.

\section{Conclusion}

In conclusion, a new statistical index, the percent CTV coverage probability (\%CCP) was successfully developed to replace the traditional CTV coverage probability for evaluating the CTV coverage loss of brain SRS/fSRS when the PTV margin is zero. Unlike the CTV coverage probability that is a degenerate function (i.e., always 0 ) when the PTV margin is 0 , the proposed percent CTV coverage probability provides a reasonable coverage probability between 0 and 1 depending on the CTV size. To calculate this new index, a previously developed statistical model was used to analyze the CTV random motion, from which the CDF and reliability function of random variable $Q$ for measuring the CTV percent coverage was derived. The \%CCP is equal to the reliability function of $Q$ for the specified percent (e.g., 95\%) CTV coverage. The closed-form expressions of $Q$ and the \%CCP 
were derived for spherical CTV and PTV using the relation of sphere-sphere intersection. Based on the calculated numerical values, it is predicted that when the PTV margin is zero, there is a significant chance $(\sim 26 \%)$ that the treatment might miss the target completely if the CTV is small ( $1 \mathrm{~mm}$ in diameter or smaller). In addition, the $95 \%$ - $95 \%$ clinical goal "at least $95 \%$ of the CTV covered by the prescription dose $95 \%$ of the time" cannot be achieved for small targets $\left(d_{\text {СTV }}<\right.$ $42 \mathrm{~mm}$ ). For the single-isocenter for multiple-targets technique, the \%CCP decreases with increasing distance between the CTV and iso-center, $d_{I \Leftrightarrow T}$. This work provided a quantitative estimate of CTV coverage loss for brain SRS/fSRS procedures that traditionally ignore this coverage loss due to the lack of proper modeling and/or blind belief of the accuracy of immobilization devices and delivery systems.

\section{Conflicts of Interest}

The author declares no conflicts of interest regarding the publication of this paper.

\section{References}

[1] Brown, R.A., Roberts, T.S. and Osborn, A.G. (1980) Stereotaxic Frame and Computer Software for CT-Directed Neurosurgical Localization. Investigative Radiology, 15, 308312. https://doi.org/10.1097/00004424-198007000-00006

[2] Leksell, L. and Jernberg, B. (1980) Stereotaxis and Tomography a Technical Note. Acta Neurochirurgica, 52, 1-7. https://doi.org/10.1007/BF01400939

[3] Jaffray, D.A. (2007) Image-Guided Radiation Therapy: From Concept to Practice. Seminars in Radiation Oncology, 17, 243-244. https://doi.org/10.1016/j.semradonc.2007.08.001

[4] Jin, J.-Y., Yin, F.-F., Tenn, S.E., Medin, P.M. and Solberg, T.D. (2008) Use of the BrainLAB ExacTrac X-Ray 6D System in Image-Guided Radiotherapy. Medical Dosimetry, 33, 124-134. https://doi.org/10.1016/j.meddos.2008.02.005

[5] Yin, F.-F., Das, S., Kirkpatrick, J., Oldham, M., Wang, Z. and Zhou, S.-M. (2006) Physics and Imaging for Targeting of Oligometastases. Seminars in Radiation Oncology, 16, 85-101. https://doi.org/10.1016/j.semradonc.2005.12.004

[6] Cernica, G., Wang, Z., Malhotra, H., De Boer, S. and Podgorsak, M.B. (2006) Investigation of Gamma Knife Image Registration Errors Resulting from Misalignment between the Patient and the Imaging Axis. Medical Physics, 33, 941-943. https://doi.org/10.1118/1.2179751

[7] Winston, K.R. and Lutz, W. (1988) Linear Accelerator as a Neurosurgical Tool for Stereotactic Radiosurgery. Neurosurgery, 22, 454-464.

https://doi.org/10.1227/00006123-198803000-00002

[8] Yeung, D., Palta, J., Fontanesi, J. and Kun, L. (1994) Systematic Analysis of Errors in Target Localization and Treatment Delivery in Stereotactic Radiosurgery (SRS). International Journal of Radiation Oncology Biology Physics, 28, 493-498. https://doi.org/10.1016/0360-3016(94)90076-0

[9] Li, G., Ballangrud, Å., Kuo, L.C., Kang, H., Kirov, A., Lovelock, M., Yamada, Y., Mechalakos, J. and Amols, H. (2011) Motion Monitoring for Cranial Frameless Stereotactic Radiosurgery Using Video-Based Three-Dimensional Optical Surface Imaging. Medical Physics, 38, 3981-3994. https://doi.org/10.1118/1.3596526 
[10] Schell, M.C., Bova, F.J., Larson, D.A., Leavitt, D.D., Lutz, W.R., Podgorsak, E.B. and Wu, A. (1995) Stereotactic Radiosurgery: Report of Task Group 42, AAPM Radiation Therapy Committee American Institute of Physics, Woodbury. https://doi.org/10.37206/53

[11] Shaw, E., Scott, C., Souhami, L., Dinapoli, R., Kline, R., Loeffler, J. and Farnan, N. (2000) Single Dose Radiosurgical Treatment of Recurrent Previously Irradiated Primary Brain Tumors and Brain Metastases: Final Report of RTOG Protocol 90-05. International Journal of Radiation Oncology Biology Physics, 47, 291-298.

https://doi.org/10.1016/S0360-3016(99)00507-6

[12] Chang, J. (2017) A Statistical Model for Analyzing the Rotational Error of Single Isocenter for Multiple Targets Technique. Medical Physics, 44, 2115-2123. https://doi.org/10.1002/mp.12262

[13] Weisstein, E.W. (n.d.) Sphere-Sphere Intersection. Wolfram MathWorld. https://mathworld.wolfram.com/Sphere-SphereIntersection.html

[14] Pope, W.B. (2018) Chapter 7: Brain Metastases: Neuroimaging. In: Schiff, D. and van den Bent, M.J., Eds., Handbook of Clinical Neurology, Elsevier, Amsterdam, Vol. 149, 89-112. https://doi.org/10.1016/B978-0-12-811161-1.00007-4 